\title{
A Study of the Relationship between Cognitive Styles and
}

\section{Learning Strategies}

\author{
Changju Shi \\ School of Foreign Language and Cultures, Nanjing Normal University \\ 122 Ninghai Road, Nan Jing 210097, China \\ E-mail: fm6666@163.com
}

Received: March 23, 2011 Accepted: April 6, 2011 doi:10.5539/hes.v1n1p20

\begin{abstract}
This study focuses on the relationship between cognitive styles and learning strategies of 184 second-year English majors from the Foreign Language School of a university in Wuhan. In this study, quantitative data is presented. Two self-reported inventories are employed. Learning Style Survey is used to examine the learning styles of the participants and the Chinese version of Oxford's Strategy Inventory for Language Learning (SILL) is conducted to survey the subjects' learning strategies.

The results show that cognitive styles have significant influence on learners' choices of learning strategies. Synthesizing style, sharpener style, field-independent style and impulsive style of cognitive styles correlate positively almost with every strategy presented in this paper, so they turn to be the most influential cognitive styles that have an impact on learners' learning strategy choices.
\end{abstract}

Finally, implications for teaching are discussed, as are suggestions for the future researches.

Keywords: Learning styles, Cognitive style, Learning strategies

\section{Introduction}

In recent years, researches in applied linguistic field have paid more attention to learners' learning styles and learning strategies which are important factors that influence the process and outcomes of learning. "Learning styles refer to an individuals' characteristics and preferred way of gathering, interpreting, organizing and thinking about information"(Wang, 2008, p. 30).It is known that cognitive styles are an important part of learning styles. Cognitive styles, defined as "a psychological construct relating to how individuals process information"(Brown and Brailsford, 2006, p. 327), have many classifications. It once was classified into field-independent style and field-dependent style, analytic style and global style, reflective style and impulsive style, and tolerance and intolerance of ambiguity. In contrast to learning styles, learning strategies are "any set of operations, plans or routines used by learners to facilitate the obtaining, retrieval, storage and use of information" (Macaro, 2006, p. 324). Oxford (1990, 74, pp. 311-327) presented learning strategies as direct and indirect strategies depending on whether they directly involve the target language or not which is a rather popular classification. Obviously, learning style and learning strategies are different. Learning styles are unconscious learner traits, but learning strategies are specific actions taken by learners to make learning more efficient.

Distinct as they are, learning styles and learning strategies have close relationship to each other. Brown pointed out that learning strategies do not operate by themselves, but rather are directly linked to the learner's innate learning styles and other personality-related factors ( $\mathrm{Li}, 2006$, p. 68). Many researches on the relationship between learning styles and learning strategies have been carried out, for example, Ehrman and Oxford (1990, pp. 311-327)explored the relationship between learning styles and learning strategies through semi-structured interviews in a qualitative study of 20 Foreign Service Institute students. Another research investigating the relationship between language learning styles and strategies of the diarist/researcher in a naturalist setting was carried out by Carson and Longhini (2002, pp. 401-438). Besides, Littlemore (2001, pp. 241-265) related different communication strategy preferences to the holistic/analytic cognitive style dimension. Moreover, a research concerned with the relationship between language learning styles and strategies was conducted by Jie Li and Xiaoqing Qin; however, the focus of the research was put on the tertiary-level learners in China. Although previous researches are fruitful, most of them are 
concerned with non-English majors, and rarely deepen into the relationship between certain aspect of learning styles and learning strategies.

Some researchers think that learning style and cognitive style are the same, while others have different opinions. Actually, they are different. Cognitive style is "individual's habitual way of organizing and processing information" (Liu, 2008, pp. 130-131), while learning style is a broader concept. Besides information processing, "learning style also contains individual's feelings and psychological behaviors" (Wang, 2009, pp. 34-35). In this paper, cognitive style is considered to be an important part of learning style and "it has great contributions to second language acquisition"(Tang, 2009, pp. 129-130). Researches on cognitive styles and learning strategies have great significance on teaching. "On one hand, after learning students' different learning styles, teachers can adopt relative(corresponding) teaching methods and strategies; on the other hand, students can choose appropriate learning strategies if they know their own cognitive styles, which can promote their autonomy and help them become successful learners"(Ma, 2003, p. 63).

This paper carries out a research on the cognitive styles and learning strategies of 178 second-year English majors from the Foreign Language School of a university in Wuhan, and will further explore the relationship between their cognitive styles and learning strategies, aiming to find some implications for language teaching and learning. Finally, some suggestions on improving teaching will be given.

\section{Researches on the Relationship between Learning Styles and Learning Strategies}

Obviously, learning styles and learning strategies are different. Learning styles embody unconscious learner traits while learning strategies are specific behaviors selected by the learner to make learner (learning) more efficient. However, these two terms are closely related to each other. Many empirical studies have suggested that learning styles may significantly influence learners' learning strategies choices in spite of different research instruments and contexts concerned.

Ehrman and Oxford (Oxford, 1990, 74, pp. 311-327) had tried to explore the relationship between learning styles and learning strategies through semi-structured interviews. Before the qualitative study, the subjects, 20 Foreign Service Institute students, had already completed two self-reported instruments as part of the quantitative study: the MBTI-G (Myers and McCaulley 1985) for learning styles and the Strategy Inventory for Language Learning (SILL) for preferred language learning strategies. Findings of this research revealed that "for each contrasting pair of the bipolar MBTI scales, the preferred learning strategy categories were in an appropriately matched distribution" (320). The research results showed that "learners' learning styles may significantly influence their choices of language learning strategies" (321).

Another research investigating the relationship between language learning styles and strategies of the diarist/researcher in a naturalist setting was carried out by Carson and Longhini (2002, pp. 401-438). The study employed Oxford's SILL and the Style Analysis Survey (SAS) to compare categories that emerge in the diary entries. The findings indicated that "the diarist's learning strategies were often affected by her learning styles" (434). For example, the diarist, with a global learning style, always suspended bits of partly understood language till they formed a large patter.

Also, another research concerned with the relationship between language learning styles and strategies was conducted by Jie Li and Xiaoqing Qin (2006, 1, pp. 67-90). However, the focus of their research is put on the tertiary-level learners in China. The study presents two kinds of data: quantitative and qualitative. In the quantitative study, the subjects consist of 187 second-year undergraduates. Two self-reported inventories, the Chinese version version of MBTI-G and a questionnaire on the use of learning strategies adapted from O'Malley and Chamot's classification system, were used to examine the students' learning styles and learning strategies respectively. Structured interviews have been performed among six high and low achievers in the qualitative aspect of the study. Their findings show that "learning styles have a significant influence on learners' strategy choices" (86). Judging scale correlates positively with seven sets of learning strategies. It also investigated the influence of the choice of learning strategies upon learning outcomes. Compared with low achievers, "high achievers are more capable of exercising strategies that are associated with their non-preferred styles" (86). Based on the results, it was proposed that "learning styles may influence language learning outcomes through their relationship with learning strategies" (86).

Study focusing on more detailed aspects of learning styles and learning strategies has also been carried out. Littlemore (2001, pp. 241-265) related different communication strategy preferences to the holistic/analytic cognitive style dimension. Their research findings showed that holistic students used more communication strategies 
that were based on comparison, and analytic students used more strategies that involved focusing on individual features of the target item.

It is clear that these researches have very fruitful results, and it has been sufficiently proved that studies concerned both learning styles and learning strategies have great significance. We believe more detailed aspect of learning style is studied, more benefits we can get. In this paper, we will concentrate on the relationship between cognitive styles and learning strategies.

\section{Research Design}

This paper aims to find the relationship between cognitive styles and learning strategies. For this purpose, a quantitative study was carried out to obtain an overall idea about the subjects' learning styles and learning strategies. Then, we will further analyze the relationship between the subjects' cognitive styles and learning strategies.

\subsection{Subject}

The subjects are 178 second-year undergraduates from the Foreign Language School of a university in Wuhan. They all major in English. They have been majoring in English for almost two years, so they are supposed to have often employed certain learning strategies influenced by their own learning styles.

\subsection{Instruments}

Two self-reported inventories are employed to examine the subjects' learning styles and learning strategies respectively. Learning Style Survey (constructed by Cohen, with Rebecca Oxford and Julie Chi) is used to examine the learning styles of the participants. Another instrument, Oxford's Strategy Inventory for Language Learning (SILL), which has been translated into Chinese, is conducted to survey the subjects' learning strategies.

\subsubsection{Measure of Learning Styles: Learning Style Survey}

Learning Style Survey we employed is constructed by Cohen, with Rebecca Oxford and Julie Chi. It was developed with an interest in those style dimensions that seem to have the most relevance to language learning. The format of the survey and a number of the dimensions and items are drawn from Oxford's Style Analysis Suevey (1995). Other dimensions and some of the wording of items are based on the work of Ehrman and Leaver (Ehrman \& Leaver, 1997, 2003; the E\&L Questionnaire 2001). For each separate item, there are five choices ranging from "never" to "always". "The premise of the survey is that all language learners have a preference for how to learn" (Cohen, 2006, p. 9). While they may have a general sense of their preferences already, this survey can help them deepen their understanding of those preferences by comparing and contrasting 11 different learning styles which are divided into three groups---Sensory/Perceptual Learning Style, Psychological Type and Cognitive Learning Style. Since cognitive styles are an important part of learning styles, and not many researches on the relationship between cognitive styles and learning strategies have been carried out, it is meaningful to focus on the association between cognitive styles and learning strategies, and some fruitful results may be obtained. This paper will concentrate on cognitive learning style, namely, Global \& Particular style, Synthesizing \& Analytic style, Sharpener \& Leveler style, Deductive \& Inductive style, Field-Independent \& Field-Dependent style, and Impulsive \& Reflective style. Global \& Particular styles show us how the subjects receive information. Global learners enjoy getting the main idea and are comfortable communicating(can comfortably communicate) even if they do not know all the words or concepts, while particular learners need specific examples to understand fully. Synthesizing \& Analytic styles are about how the subjects further process information. Learners with synthesizing style like to find and organize key points into a summary and enjoy guessing meanings and predicting outcomes, while learners with analytic style like to think and analyze and often focus on rules and generalization. Sharpener \& Leveler styles tell us how the subjects commit material into memory. Specifically, sharpeners notice differences and distinctions among items and store items separately and retrieve them individually, while levelers usually clump material to remember it by eliminating or reducing differences and by focusing almost on similarities. Deductive \& Inductive styles illustrate how learners deal with language rules. Deductive learners like to go from general to specific and start with rules and theories rather than specific examples. In the contrary, inductive learners like to go from specific to general and begin with examples rather than rules or theories. Besides, Field-Independent \& Field-Dependent styles are about how participants deal with multiple inputs. Field-Independent learners are able to handle the language parts as well as the whole without being distracted, while Field-Dependent learners need context to focus on and understand something and may take in language one part at a time. Last but not least, Impulsive \& Reflective styles have something to do with how learners'(learners) dealing with response time. Generally, with impulsive style, learners would react 
quickly in acting or speaking without thinking the situation thoroughly, while reflective learners process information at a low speed with high accuracy.

\subsubsection{Measure of Learning Strategies Strategy Inventory for Language Learning}

Oxford's Strategy Inventory for Language Learning is employed to measure subjects' learning strategies. We adopt a Chinese version of it, and it contains 50 items which are divided into 6 kinds of learning strategies, namely memory strategy, cognitive strategy, compensation strategy, metacognitive strategy, affective strategy and social strategy. For each statement of the 50 items, there are five choices ranging from "never or almost never" to "always or almost always".

\subsubsection{Data Collection}

The subjects finished the two questionnaires on learning styles and learning strategies in class. Before the participants started to response to the questionnaires, the researcher gave them detailed instructions and they were told that they could ask questions in the process if there was anything they did not understand. Also, they were told that all the results would be applied to research work only and their responses would have nothing to do with teacher's evaluation of them. Furthermore, the participants were informed that they could leave the classroom anytime(at any time) they liked. In the final analysis, 170 questionnaires were considered valid.

\subsubsection{Data Analysis}

Data of cognitive styles and learning strategies were computed separately with the help of Excel. Then the Statistical Package of Social Science SPSS was employed to compute the data gained in the study. The statistical procedure of stepwise multiple regression was the main analysis for testing the relationship between cognitive styles and learning strategies.

\section{Findings and Discussion}

The stepwise multiple regression was used to study the relationship between cognitive styles and learning strategies. In this research, cognitive styles were regarded as the independent variables, and the participants' learning strategy choices were dependent variables. As was mentioned earlier, each pair of the dimensions of cognitive style appeared to be in opposition (i.e. global \& particular style, synthesizing \& analytic style, sharpener \& leveler style, deductive $\&$ inductive style, field-independent $\&$ field-dependent style, and impulsive $\&$ reflective style), so a high score on one might indicate a low score on the other, which meant that scores on cognitive styles were continuous. Therefore, in this study, scores of first one of each pair (i.e., global, synthesizing, sharpener, deductive, field-independent, and impulsive) were treated as independent variables in the regression analysis. The results of the relationship between cognitive styles and each of the six learning strategies were synthesized into Table 1.

Among the six pairs of cognitive styles, synthesizing style, and impulsive style were found to have significant influence on the choice of learning strategies, namely the memory strategies of grouping and imagery, the cognitive strategies of practicing, analyzing and summarizing, the compensation strategies of guessing, the metacognitive strategies of planning, paying attention and self-evaluating, the affective strategies of anxiety-reduction and self-encouraging and the social strategies of cooperation, turning out to be the most influential styles in present study. Field-Independent style associates positively with all the learning strategies mentioned above except compensation strategies, and sharpener style with all strategies except compensation strategy. Moreover, deductive learners tend to have a preference for cognitive learning strategies.

Deep analysis of the results shows that a particular cognitive style except global style is always positively related to certain strategies. For instance, synthesizing learners show likings for cognitive strategy $(\mathrm{p}<.01)$, compensation strategy $(p<.01)$, metacognitive strategy $(p<.01)$, affective strategy $(p<.05)$ and social strategy $(p<.01)$; field-independent learners tend to employ memory strategy $(p<.01)$ and cognitive strategy $(p<.01)$ more frequently, and use metacognitive strategy $(\mathrm{p}<.01)$, affective strategy $(\mathrm{p}<.01)$ and social strategy $(\mathrm{p}<.01)$ as well. This is consistent with the results of some previous researches that examined the relationship between learning styles and learning strategies and reported that learning styles may significantly influence learners' choice of learning strategies.

Theoretically, synthesizing learners tend to find and organize key points into a summary, enjoy guessing meaning and outcomes predictios and notice similarities quickly. If so, synthesizing learners show a preference for cognitive strategies which include reasoning, analyzing, summarizing and practicing in this way. Specifically, when reading a passage, subjects tend to first skim it over to get the main idea, then go back to read carefully, and they tend to find fixed English patterns while studying as illustrated in this study. Since they enjoy guessing meaning and outcome 
predictions, they would apply the strategy of anxiety-reduction and imagery more frequently to retrieve information and relax. Subjects in this study make guesses by putting the unfamiliar English words in the context to understand them, and try to guess what the other person will say next in English. In the whole process, synthesizing learners are supposed to ask questions for necessary clarification so as to facilitate their meaning guessing and outcome predictions.

As to field-independent learners, they are able to handle the language parts as well as the whole without being distracted. Learners should focus on special parts of learning tasks so as to handle language parts. Also, self-encouragement and anxiety-reduction which belong to affective strategies would help them not to be easily distracted. A better understanding of English culture, as part of social strategies selected by learners in the present study, can help them improve their understanding and enhance their production of the target language, and process the whole of the learning materials. Since they can deal with language parts well, it is usual for them to connect learning tasks with other similar things to remember them. As is revealed in the present study, students often facilitate their memorization of new words with the help of relative images and gestures. Moreover, the more learners know about a language or a culture, the more desirable the language or culture would be to them, so learners might do a lot of practice which is included in the cognitive strategies to enhance their learning, which will be in turn beneficial to the development of field-independent style, for "strategies are not totally fixed" (Bostrom, 2006, p. 183).

With impulsive style, learners would react quickly in acting or speaking without thinking the situation thoroughly. In order to process information at high speed, they tend to use memory strategies which include grouping and imagery in the present study very often so as to grasp and store message quickly. Moreover, with likings for reacting quickly, it is not unusual for impulsive learners do a lot of practice through talking in English, or watching English movies and TV programs in their study as revealed in the present research. Also, because of their haste in speaking or acting, impulsive learners need to employ compensation strategies including guessing meaning and using gestures, coining words and synonyms to keep the communication going on. Actually, in the process of guessing meanings, they also apply anxiety-reduction strategy which belongs to affective strategy. Given their characteristics, it is inevitable for impulsive learners to make some mistakes. In this case, they, with their courage, would ask others to help them correct their mistakes, which indicates they might have good cooperation with others, such as practicing English with others.

Generally, sharpeners are inclined to notice differences and distinctions among items while committing material to memory. Besides, they store items separately and retrieve them individually. This means when they are learning new learning materials, they will pay selective attention to the learning materials and try to analyze and summarize them so as to find the differences quickly. In the present study, it is revealed through subjects' choice of metacognitive strategies of noticing people's wording when speaking, being aware of one's own learning for they do a lot of planning, reading, listening, and cognitive strategies of summarizing sentence patterns and abstracting stories, etc. Moreover, sharpeners will recall the relative knowledge stored earlier to facilitate memorization of new learning task, and they are actually using memory strategies which, in the present study, include relating the sound of English words to some images to facilitate memorization, memorizing English words with the help of the position they once appeared, etc. Sharpeners store items separately. So when they cannot recall a word, they can use a similar word to replace it. Actually, they are using compensation strategies as they do so. Accordingly, they must encourage themselves to reduce anxiety in this process.

In this study, deductive style was found to have certain positive relationship with cognitive strategies. However, no relationship was identified between deductive style and every item of cognitive strategies. It is presumed that statistics of every item of cognitive strategies might be in dispersion, so although deductive style was found to have some relationship with cognitive strategies on the whole, no obvious relationship exist between deductive style and every item of cognitive strategies.

As is shown above that synthesizing style, sharpener style, field-independent style and impulsive style of cognitive styles have significant relationship with learner's use of learning strategies, so cognitive styles are definitely an important part of learning styles.

\section{Conclusion}

The major findings, details of which have been discussed in section four of this paper, on the relationship between cognitive styles and learning strategies are listed below:

(i) Cognitive styles have a significant influence on learners' choices of learning strategies. 
(ii) Synthesizing style, sharpener style, field-independent style and impulsive style of cognitive styles correlate positively almost with every strategy presented in this paper, so they turn to be the most influential cognitive styles that have an impact on learners' learning strategy choices.

This kind of study is valuable to students. Each style preference offers significant strengths in learning and working. As a result, if students can recognize their strengths, they can take advantage of ways they learn best. Moreover, by being aware of the style areas they do not use, then developing them, students can enhance their learning and working power. Dealing with tasks that do not seem quite suited to students style preferences will help them stretch beyond their comfort zone and expand their learning and working potential, which is definitely beneficial to students themselves.

Furthermore, it is not useless for teachers, either. "Strategies are neither totally fixed nor flexible and teachers can both build on existing strengths and develop additional competencies for their students" (Bostrom, 2006, p. 184), so teachers should pay attention to students' learning styles in their teaching, and adopt relative teaching methods that are consistent with most students' learning styles. Cognitive styles are closely connected with students' learning strategies as is revealed in the present study, so in the classroom, there should be training concerned with learning styles and learning strategies. Some students might have been aware of their own learning styles, but they might not apply correlated learning strategies for better language learning. One of the aims of strategy training is to change students' attitude towards their learning ability, so they can understand their failure in study is not due to their lack of aptitude rather than their lack of effective learning strategies. As a result, teachers should try to understand students' comments on their own learning and use of strategies. In this way, students can raise their awareness of learning strategies.

This paper did not study the influence of learning strategy choices on learning outcomes. Future study should investigate whether students' cognitive styles have impact on learners' learning outcomes. It is inferred that students' cognitive styles have positive connection with their learning outcomes through the interaction of cognitive styles and learning strategies, for the research conducted by Jie Li and Xiaoqing Qin showed that the choice of learning strategies had influence upon learning outcomes. Compared with low achievers, high achievers are more capable of exercising strategies that are associated with their non-preferred styles. However, their study was concerned with all the learning styles and did not pay special attention to cognitive styles. As a result, further research related to the influence of cognitive style on learning outcomes need to be carried out. Maybe, some meaningful results will be found.

In the end, since this study is on the basis of a sample of second-year English majors in one university, it is just suggestive, for different learning environment may influence students' learning. Also, a much wider research that is concerned with more English majors around China should be carried out to gain much more representative findings.

\section{References}

Boström, L., Liv M., \& Lassen. (2006). Unraveling learning, learning styles, learning strategies and meta-cognition, Education + Training, 48, 178-189, doi:10.1108/00400910610651809, http://dx.doi.org/10.1108/00400910610651809

Brown, Elizabeth, et al, (2006), Reappraising Cognitive Styles in Adaptive Web Applications. [Online] Available: http://portal.acm.org/citation.cfm?id=1135827

Carson, J. G. \& Longhini, A. (2002), Focusing on learning styles and strategies: A diary study in an immersion setting. Language Learning, 52, 401-438, doi:10.1111/0023-8333.00188, http://dx.doi.org/10.1111/0023-8333.00188

Cohen, Andrew D., \& Weaver, Susan J. (2006). Styles- and strategies- Based Instruction: A Teachers' Guide. Bei Jing: Foreign Language Teaching and Research Press.

Ehrman, M., \& Oxford,R. (1990). Adult language learning styles and strategies in an inventive training setting. Modern Language Journal, 74, 311-327

Li Jie \& Qin Xiaoqing. (2006). Language learning styles and learning strategies of tertiary-level English learners in China. Regional Language Center Journal, 37, 367-390, doi: 10.1177/0033688206063475, http://dx.doi.org/10.1177/0033688206063475

Littlemore, Jeannette. (2001). An empirical study of the relationship between cognitive style and the use of communication strategy. Applied Linguistics, 22, 241-265, doi:10.1093/applin/22.2.241, http://dx.doi.org/10.1093/applin/22.2.241 
Liu Chang. (2008). Research on theories of cognitive styles. Time Education, 5, 130-131

Macaro, E. (2006), Strategies for language learning and for language use: Revising the Theoretical Framework. The Modern Language Journal, 90, 320-337, doi:10.1111/j.1540-4781.2006.00425.x, http://dx.doi.org/10.1111/j.1540-4781.2006.00425.x

Ma Yaju. (2008). On cognitive style and selection of teaching strategies. Journal of Weinan Teachers College, 23(3), 63

Tang Jing. (2009). Cognitive style has an effect on learning strategy. Journal of Hubei Radio \&Television University. 29(2), 129-130

Wang Mingli. (2008). Learning styles and English teaching. US-China Foreign Language, 6, 30.

Wang Xuemei. (2009). Cognitive styles and English postgraduates academic competence development. Shandong Foreign Language Teaching Journal. 1, 34-35

\section{Appendix 1}

Table1. Relationship between Cognitive Styles and Learning Strategies

\begin{tabular}{|c|c|c|c|c|c|c|c|}
\hline & & Memory & Cognitive & Compensation & Meta-cognitive & Affective & Social \\
\hline \multirow{3}{*}{ Global } & $\mathrm{P}$ & -.014 & -.017 & .046 & .015 & -.042 & .078 \\
\hline & $\mathrm{S}$ & .851 & .823 & .555 & .842 & .589 & .314 \\
\hline & $\mathrm{N}$ & 170 & 170 & 170 & 170 & 170 & 170 \\
\hline \multirow{3}{*}{ Synthesizing } & $\mathrm{P}$ & $.181 *$ & $.376^{* *}$ & $.329 * *$ & $.343 * *$ & $.249 *$ & $.256 * *$ \\
\hline & $\mathrm{S}$ & .018 & .000 & .000 & .000 & .001 & .001 \\
\hline & $\mathrm{N}$ & 170 & 170 & 170 & 170 & 170 & 170 \\
\hline \multirow{3}{*}{ Sharpener } & $\mathrm{P}$ & $.369 * *$ & $.354 * *$ & .199 & $.468 * *$ & $.271 * *$ & $.241 * *$ \\
\hline & $\mathrm{S}$ & .000 & .000 & .009 & .000 & .000 & .002 \\
\hline & $\mathrm{N}$ & 170 & 170 & 170 & 170 & 170 & 170 \\
\hline \multirow{3}{*}{ Deductive } & $\mathrm{P}$ & -.041 & $.158 *$ & .040 & .090 & .022 & -.020 \\
\hline & $\mathrm{S}$ & .591 & .039 & .601 & .241 & .778 & .799 \\
\hline & $\mathrm{N}$ & 170 & 170 & 170 & 170 & 170 & 170 \\
\hline \multirow{3}{*}{ Field-independent } & $\mathrm{P}$ & $.300 * *$ & $.384 * *$ & .128 & $.359 * *$ & $.270 * *$ & $.241 * *$ \\
\hline & $\mathrm{S}$ & .000 & .000 & .097 & .000 & .000 & .002 \\
\hline & $\mathrm{N}$ & 170 & 170 & 170 & 170 & 170 & 170 \\
\hline \multirow{3}{*}{ Impulsive } & $\mathrm{P}$ & $.232 *$ & $.285 * *$ & $.316^{* *}$ & $.299 * *$ & $.226 * *$ & $.226^{* *}$ \\
\hline & $\mathrm{S}$ & .002 & .000 & .000 & .000 & .003 & .003 \\
\hline & $\mathrm{N}$ & 170 & 170 & 170 & 170 & 170 & 170 \\
\hline
\end{tabular}

\footnotetext{
* Correlation is significant at the 0.05 level (2-tailed).

** Correlation is significant at the 0.01 level (2-tailed).

$\mathrm{P}=$ Pearson Correlation, $\mathrm{S}=$ sig. (2-tailed), $\mathrm{N}=$ Number.
} 